\title{
Remark to the Ergodic Decomposition of Measures
}

By

\author{
Hiroaki SHImomuRA*
}

\section{$\S 1$. Introduction}

Let $(X, \mathfrak{B}, \mu)$ be a measure space and $\mathfrak{X}$ be a sub- $\sigma$-field of $\mathfrak{B}$. A family $\left\{\mu^{x}\right\}_{x \in X}$ of probability measures on $\mathfrak{B}$, indexed by $x$ is called a system of conditional probabilities with respect to $\mathfrak{X}$ or a disintegration of $\mu$ with respect to $\mathfrak{U}$ if it has the following properties, namely

(a) $\forall B \in \mathfrak{B}$, the function $x \mapsto \mu^{x}(B)$ is $\mathfrak{A}$-measurable and

(b) $\forall_{B \in \mathfrak{B},}^{\forall} A \in \mathfrak{A}, \quad \mu(B \cap A)=\int_{A} \mu^{x}(B) d \mu(x)$.

In general, disintegrations of $\mu$ with respect to $\mathfrak{X}$ do not exist. (See an example in the later discussions.) However, if $(X, \mathfrak{B})$ is standard (that is, the measurable space $(X, \mathfrak{B})$ is isomorphic to $\left(Y, \mathfrak{B}_{Y}\right)$, where $Y$ is a Polish space and $\mathfrak{B}_{Y}$ is the Borel $\sigma$-field of $Y$ ), then a disintegration of any probability measures on $\mathfrak{B}$ exists for all $\mathfrak{X}(\subset \mathfrak{B})$. (For example, see [1].) If a disintegration of $\mu$ with respect to $\mathfrak{X}$ exists, then for any fixed $A \in \mathfrak{Q}, \mu^{x}(A)=\chi_{A}(x)$ holds for $\mu$-a.e.x, where $\chi_{A}$ is the indicator function of $A$. Especially for any fixed $A \in \mathfrak{A}, \mu^{x}(A)$ takes only the values 0 or 1 for $\mu$-a.e.x. A strengthening form of this result is as follows.

(c) For $\mu$-a.e.x, $\mu^{x}$ takes only the values 0 or 1 on $\mathfrak{A}$.

If a disintegration $\left\{\mu^{x}\right\}_{x \in X}$ of $\mu$ with respect to $\mathscr{U}$ satisfies (c), then it is called an ergodic decomposition. The following fact is known for the ergodic decomposition.

Theorem. Let $(X, \mathfrak{B})$ be a standard space, $\left\{\mathfrak{U}_{n}\right\}(n=1,2, \cdots)$ be a decreasing

Communicated by S. Matsuura, March 1, 1990. Revised April 9, 1990.

* Department of Mathematics, Fukui University, Fukui 910, Japan. 
sequence of countably generated sub- $\sigma$-fields of $\mathfrak{B}$ and $\mathfrak{A}=\bigcap_{n=1}^{\infty} \mathfrak{A}_{n}$. Then for any probability measure $\mu$ on $\mathfrak{B}$, the disintegration of $\mu$ with respect to $\mathfrak{A}$ is ergodic.

For the proof, see [2] or [3].

However even in a standard space, taking a suitable sub- $\sigma$-field $\mathfrak{\Re}$ there does exist a probability measure whose disintegration with respect to $\mathscr{N}$ is non ergodic. The purpose of this note is to give such an example.

\section{§2. Examples}

Let $\mathbb{R}^{\infty}$ be the countable direct product of $\mathbb{R}, \mathfrak{B}\left(\mathbb{R}^{\infty}\right)$ be the Borel $\sigma$-field on $\mathbb{R}^{\infty}$ and $\lambda$ be the standard Lebesgue measure on $(0,1]$. Take $0<s<1 / 2$, and using indicator function $\chi_{n, k}(\tau)$ of the intervals $((k-1) / n, k / n](n=1,2, \cdots$, $k=1,2, \cdots, n)$ define a map $\phi(\tau)=\left(\phi_{h}(\tau)\right)_{h}$ from $(0,1]$ to $\mathbb{R}^{\infty}$ such that $\phi_{h}(\tau)=$ $\left(n^{s} \chi_{n, k}(\tau)+1\right) \sqrt{\tau}$, if $h=2^{-1} n(n-1)+k(1 \leqq k \leqq n)$. Then,

$$
\int_{0}^{1} \phi_{k}(\tau)^{2} d \lambda(\tau) \leqq 2\left(n^{2 s} / n+1\right) \leqq 4 .
$$

Hence for all $a=\left(a_{h}\right)_{h} \in l^{2}$, we have

$$
\sum_{h=1}^{\infty} a_{h}^{2} \phi_{h}^{2}(\tau)<\infty \quad \text { for } \lambda \text {-a.e. } \tau_{\text {。 }}
$$

However $\left\{\phi_{h}(\tau)\right\}_{h}$ is not bounded for each $\tau \in(0,1]$, so

$$
{ }^{\forall} \in(0,1],{ }^{\exists} b=\left(b_{h}\right)_{h} \in l^{2}, \text { s.t., } \sum_{h=1}^{\infty} b_{h}^{2} \phi_{h}^{2}(\tau)=\infty 。
$$

Now let $g$ be the standard Gaussian measure with mean 0 and variance 1 on the usual Borel field $\mathfrak{B}(\mathbb{R}), d g(t)=(2 \pi t)^{-1 / 2} \exp \left(-2^{-1} t^{2}\right) d t$ and $G$ be the product measure of $g, G=\mathbb{I}_{h=1}^{\infty} g$. Using transformations $T_{\tau}, S_{\tau}$ on $\mathbb{R}^{\infty}, T_{\tau}: x=\left(x_{h}\right) \in$ $\mathbb{R}^{\infty} \mapsto\left(\phi_{h}(\tau) x_{h}\right) \in \mathbb{R}^{\infty}, S_{\tau}: x=\left(x_{k}\right) \in \mathbb{R}^{\infty} \mapsto \sqrt{\tau}\left(x_{h}\right) \in \mathbb{R}^{\infty}$, we put $T_{\tau} G=G^{\tau}, S_{\tau} G$ $=G_{\tau}$ and $\mu^{\tau}=2^{-1}\left(G^{\tau}+G_{\tau}\right)$. Then since

(4) $\sum_{h=1}^{\infty} a_{h}^{2} x_{h}^{2}<\infty$ holds for $G$-a.e.x $=\left(x_{h}\right)_{h}$ if and only if $a=\left(a_{h}\right)_{h} \in l^{2}$, we have for the spaces $H_{a}=\left\{x=\left(x_{h}\right)_{h} \in \mathbb{R}^{\infty} \mid \sum_{h=1}^{\infty} a_{h}^{2} x_{h}^{2}<\infty\right\}$ indexed by $a=$ $\left(a_{k}\right)_{h} \in l^{2}$,

(5) $\mu^{\tau}\left(H_{a}\right)=1$ if $\sum_{h=1}^{\infty} a_{h}^{2} \phi_{h}^{2}(\tau)<\infty$, and $\mu^{\tau}\left(H_{a}\right)=1 / 2$ if $\sum_{h=1}^{\infty} a_{h}^{2} \phi_{h}^{2}(\tau)=\infty$ 。

Next take $\tau \in(0,1]$ and fix it. Then for each $n$ there exists unique $1 \leqq k_{n} \leqq n$ 
which satisfies $\chi_{n, k_{n}}(\tau)=1$. Put $h_{n}=2^{-1} n(n-1)+k_{n}$. Then in virtue of the law of large numbers,

$$
\lim _{n \rightarrow \infty} \frac{x_{1}^{2}+\cdots+x_{2^{-1}}^{2} n(n-1)}{2^{-1} n(n-1)}=1 \quad \text { for } G \text {-a.e.x and }
$$

$$
\lim _{n \rightarrow \infty} \frac{x_{h_{1}}^{2}+\cdots+x_{h_{n}}^{2}}{n-1}=1 \quad \text { for } G \text {-a.e.x. }
$$

Consequently, it follows from $2 s<1$,

$$
\lim _{n \rightarrow \infty} \frac{x_{h_{1}}^{2}+\cdots+x_{h_{n}}^{2}}{2^{-1} n(n-1)}=0 \quad \text { for } G^{\tau} \text {-a.e.x. }
$$

Hence,

$$
\lim _{n \rightarrow \infty} \frac{x_{1}^{2}+\cdots+x_{2}^{2} n(n-1)}{2^{-1} n(n-1)}=\tau \quad \text { for } G^{\tau} \text {-a.e.x. }
$$

On the other hand, it is easy to see that

$$
\lim _{n \rightarrow \infty} \frac{x_{1}^{2}+\cdots+x_{2}^{2}-1 n(n-1)}{2^{-1} n(n-1)}=\tau \quad \text { for } G_{\tau} \text {-a.e.x. }
$$

Thus we have,

$$
\lim _{n \rightarrow \infty} \frac{x_{1}^{2}+\cdots+x_{2}^{2}-1 n(n-1)}{2^{-1} n(n-1)}=\tau \quad \text { for } \quad \mu^{\tau} \text {-a.e.x. }
$$

Define $p(x)=\lim _{n \rightarrow \infty} \frac{x_{1}^{2}+\cdots+x_{2}^{2}-1 n(n-1)}{2^{-1} n(n-1)}$, if the limit exists and $p(x)=0$, otherwise. Then it follows from (11) that $p(x)=\tau$ for $\mu^{\tau}$-a.e.x and

$$
\mu^{\tau}\left(p^{-1}(E)\right)=\chi_{E}(\tau) \text { holds for all } E \in \mathfrak{B}(\boldsymbol{R}) .
$$

Now put $\mu(B)=\int_{0}^{1} \mu^{\tau}(B) d \lambda(\tau)$ for $B \in \mathfrak{B}\left(\boldsymbol{R}^{\infty}\right)$. Then for all $B \in \mathfrak{B}\left(\boldsymbol{R}^{\infty}\right)$ and for all $E \in \mathfrak{B}(\boldsymbol{R})$ we have $\mu\left(B \cap p^{-1}(E)\right)=\int_{E} \mu^{\tau}(B) d \lambda(\tau)$. Especially,

$$
p \mu=\lambda
$$

and

$$
\mu\left(B \cap p^{-1}(E)\right)=\int_{p^{-1}(E)} \mu^{p(x)}(B) d \mu(x) .
$$

Further from (2) and (5) we have $\mu^{\tau}\left(H_{a}\right)=1$ for $\lambda$-a.e. $\tau$ and therefore $\mu\left(H_{a}\right)=1$. Thus,

(15) $\mu\left(B \cap H_{a}\right)=\int_{H_{a}} \mu^{p(x)}(B) d \mu(x)$ for all $B \in \mathfrak{B}\left(\boldsymbol{R}^{\infty}\right)$ and for all $a=$ $\left(a_{k}\right)_{k} \in l^{2}$. 
Let $\mathscr{Q}$ be a $\sigma$-field generated by $p^{-1}(\mathfrak{B}(\mathbb{R}))$ and $H_{a}\left(a \in l^{2}\right)$. Then it is easy to see that

(16) for a fixed $B \in \mathfrak{B}\left(\boldsymbol{R}^{\infty}\right), \mu^{p(x)}(B)$ is an $\mathfrak{X}$-measurable function of $x$ and

(17) $\mu(B \cap A)=\int_{A} \mu^{p(x)}(B) d \mu(x)$ for all $B \in \mathfrak{B}\left(\mathbb{R}^{\infty}\right)$ and for all $A \in \mathfrak{Q}$.

From (16) and (17) it follows that $\left\{\mu^{p(x)}\right\}_{x \in R^{\infty}}$ is the disintegration of $\mu$ with respect to 2 . However for any $\tau$ there exists $b=\left(b_{h}\right)_{h} \in l^{2}$ which has property stated in (3). Consequently, $\mu^{\tau}\left(H_{b}\right)=1 / 2$ and therefore $\left\{\mu^{p(x)}\right\}_{x \in \mathbb{R}^{\infty}}$ is non ergodic decomposition.

Finally we will give a simple example of $(X, \mathfrak{B})$ on which a probability measure $\mu$ does not admit any disintegration with respect to a sub- $\sigma$-field $\mathfrak{A}$.

Let $X=[0,1]$, and consider a probability measure $\mu$ on $\mathfrak{B}([0,1])$ without atomic part. Let $\mathfrak{A}=\mathfrak{B}([0,1])$ and let $\mathfrak{B}$ be the $\sigma$-field of all $\mu$-measurable sets. Suppose that there would exist some disintegration $\left\{\mu^{x}\right\}_{x \in X}$ of $\mu$. Then for each $A \in \mathfrak{A}, \mu^{x}(A)=\chi_{A}(x)$ holds for $\mu$-a.e.x. Since $\mathfrak{A}$ is countably generated, there exists $\Omega \in \mathfrak{Y}$ with $\mu(\Omega)=1$ such that $x \in \Omega$ implies $\mu^{x}=\delta_{x}$ on $\mathfrak{Q}$, where $\delta_{x}$ is the Dirac measure at $x$. Especially we have $\mu^{x}(\{x\})=1$ for all $x \in \Omega$. Hence it holds $\mu^{x}=\delta_{x}$ on $\mathfrak{B}$ for all $x \in \Omega$. Take any $B \in \mathfrak{B}$ and put $C=\left\{x \in \mathbb{R} \mid \mu^{x}(B)\right.$ $=1\}$. Then $C \in \mathfrak{Y}$ and $C \cap \Omega=B \cap \Omega$. Thus we have $B \cap \Omega \in \mathfrak{A}$ for all $B \in \mathfrak{B}$. By the way the following lemma shows that there exists $N \in \mathfrak{Q}$ such that $N \subset \Omega$, ${ }^{\sharp} N=\aleph$ and $\mu(N)=0$. It follows from these facts that $" \mathfrak{Q}=2^{*}$. But it contradicts to $\mathbb{Q}=\mathbb{N}$, since $\mathfrak{Q}$ is countably generated.

Lemma. Let $\mu$ be a probability measure on $\mathfrak{B}([0,1])$ without atomic part and $\Omega$ be a $\mu$-measurable set with $\mu(\Omega)>0$. Then there exists Borel subset $N$ of $\Omega$ such that $N=\aleph$ and $\mu(N)=0$.

Proof. Without loss of generality we may assume that $\Omega$ is a compact subset of $[0,1]$. Put $f(t)=\mu(\Omega)^{-1} \mu(\Omega \cap[0, t])$ for $0 \leqq t \leqq 1$. By the assumption $f$ is continuous and it is easily checked that

$$
\mu\left(\Omega \cap f^{-1}([\alpha, \beta])\right)=(\beta-\alpha) \mu(\Omega) \quad \text { for } \quad 0 \leqq \forall \alpha \leqq \forall \beta 1 .
$$

Hence we have

$$
\mu\left(\Omega \cap f^{-1}(E)\right)=\mu(\Omega) \lambda(E) \quad \text { for all } E \in \mathfrak{B}([0,1]) .
$$

It follows from (18) that $\Omega \cap f^{-1}([\alpha, \beta]) \neq \emptyset$ for $0 \leqq \forall \alpha \leqq \forall \beta \leqq 1$. So using the complete intersection property of compact sets, $\Omega \cap f^{-1}(\alpha) \neq \emptyset$ holds for all 
$\alpha \in[0,1]$. Now take Cantor's ternary set $C$ and put $N=\Omega \cap f^{-1}(C)$. Then $\mu(N)=0$ holds by (19) and $N=N$ holds by the above arguments. Q.E.D.

\section{References}

[1] Parthasarathy, K.R., Probability measures on metric spaces, Academic Press, 1967.

[2] Shimomura, H., Ergodic decomposition of quasi-invariant measures, PUBL. RIMS, Kyoto Univ., 14 (1978), 359-381.

[3] Schwartz, L., Lectures on disintegration of measures, Tata Institute of Fundamental Research, 1976. 
